Femtosecond laser machining of multi-depth microchannel networks onto silicon

This article has been downloaded from IOPscience. Please scroll down to see the full text article.

2011 J. Micromech. Microeng. 21045027

(http://iopscience.iop.org/0960-1317/21/4/045027)

View the table of contents for this issue, or go to the journal homepage for more

Download details:

IP Address: 141.211.173.82

The article was downloaded on 06/04/2012 at 16:52

Please note that terms and conditions apply. 


\title{
Femtosecond laser machining of multi-depth microchannel networks onto silicon
}

\author{
D H Kam ${ }^{1}$, L Shah ${ }^{2,3}$ and J Mazumder ${ }^{1}$ \\ ${ }^{1}$ Center for Lasers and Plasmas in Advanced Manufacturing, University of Michigan at Ann Arbor, \\ MI 48109, USA \\ 2 IMRA America Inc., Ann Arbor, MI 48105, USA \\ E-mail: mazumder@umich.edu
}

Received 15 November 2010, in final form 18 January 2011

Published 24 March 2011

Online at stacks.iop.org/JMM/21/045027

\begin{abstract}
Direct writing of multi-depth microchannel branching networks into a silicon wafer with femtosecond pulses at $200 \mathrm{kHz}$ is reported. The silicon wafer with the microchannels is used as the mold for rapid prototyping of microchannels on polydimethylsiloxane. The branching network is designed to serve as a gas exchanger for use in artificial lungs and bifurcates according to Murray's law. In the development of such micro-fluidic structures, processing speed, machining range with quality surface, and precision are significant considerations. The scan speed is found to be a key parameter to reduce the processing time, to expand the machining range, and to improve the surface quality. By fabricating a multi-depth branching network as an example, the utilization of femtosecond pulses in the development of microfluidic devices is demonstrated.
\end{abstract}

(Some figures in this article are in colour only in the electronic version)

\section{Introduction}

Femtosecond (fs) laser pulses have been attractive for a wide variety of micromachining applications because of their ability to fabricate micron-level features with minimal peripheral damage and associated debris. The machined quality and the resolution of such ultrashort laser pulses have been proven in many 'proof of concept' demonstrations [1-5]. However, the low productivity of fs pulses has limited their contribution in practical applications. This study investigates fs pulses with a high repetition rate $(200 \mathrm{kHz})$ to fabricate a wide range of microchannel structures onto silicon with quality surface and increased processing speed. This is demonstrated by fabricating microchannel branching networks to replicate the flow of blood in mammalian vasculature to serve gas exchangers for use in artificial lungs.

Current generation of artificial lungs is not efficient enough to support patients with chronic irreversible progressive pulmonary failure [6-9]. Structurally, the

3 Present address: Townes Laser Institute, CREOL College of Optics and Photonics, University of Central Florida, Orlando, FL 32816, USA. inefficiency of current hollow fiber blood oxygenators is attributed to their non-physiological features, which include long diffusion lengths, high flow resistances, and non-uniform flow distributions [10]. The lung-like features are essential to the design of an artificial lung to enhance the gas exchange performance while maintaining the biocompatible blood flow condition. In the natural vasculature, the trade-off between the pressure drop and the diffusion lengths of the blood vessel is well balanced with uniform flow distribution. This optimal vascular architecture follows a relationship known as Murray's law $[11,12]$. For a bifurcation, as illustrated in figure 1(a), Murray's law states that the radius of the parent vessel $\left(r_{0}\right)$ and the radii of daughter vessels $\left(r_{1}\right.$ and $\left.r_{2}\right)$ have the following relationship:

$$
r_{0}^{3}=r_{1}^{3}+r_{2}^{3} .
$$

The human pulmonary vasculature consists of 15 branching levels, with diameters ranging from $\sim 20 \mu \mathrm{m}$ up to several millimeters [13].

In order to create an efficient gas exchange and a biocompatible flow condition, we seek to replicate natural 


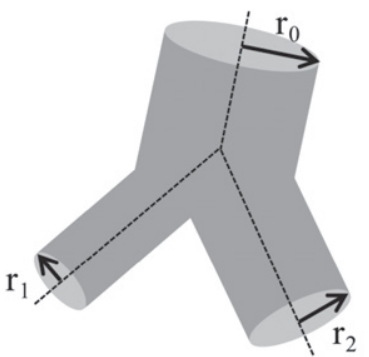

(a)

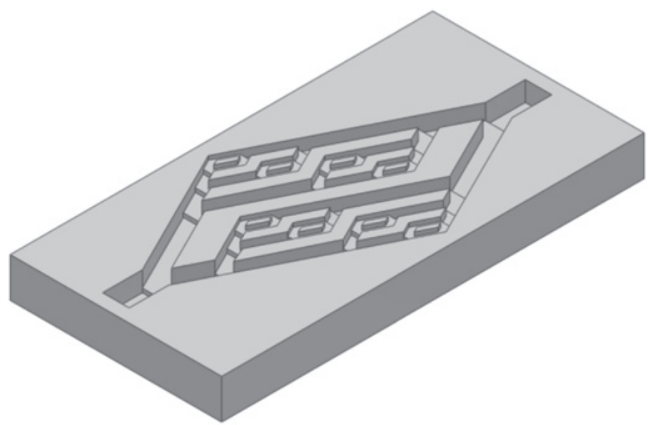

(b)

Figure 1. Schematics of $(a)$ bifurcation and $(b)$ multi-depth microchannel network.

vascular networks. Multi-depth microchannel branching networks are proposed as gas exchangers to mimic the natural vascular structure. The network bifurcates according to Murray's law and thus the channels become shallower and narrower at each bifurcation as illustrated in figure $1(b)$. To apply Murray's law to rectangular cross sections, the hydraulic diameter is used instead of the radius. Here, the aspect ratio of the width to the depth of channels in the network is designed to be constant. While single depth branching designs can implement Murray's law by varying the width of each generation [14], the multi-depth design is beneficial for a more compact design which could be easily implantable. In the development of the networks, a wide range of microchannel size with reasonable surface quality and resolution is necessary to realize various designs. The current phase of our artificial lung development requires multilevel structures with depths ranging from $\sim 20 \mu \mathrm{m}$ to several hundreds of microns. Additionally, the surface roughness should be minimized to reduce any adverse effect of surface roughness which may initiate blood clotting and clogging $[6,10]$.

This study describes fs laser machining of multidepth microchannel networks into silicon as a part of the development of artificial lungs. It is possible to build such branching networks using lithography and etching-based techniques; however, the mask-based lithographic techniques are inefficient in terms of cost and development time, and it is difficult to lithographically vary the depth of such multi-depth structures. An alternative method to reduce cost and time is the laser direct writing, which does not need any masks. In addition, the flexibility of the laser process facilitates creating multi-depth structures of such branching networks. In our previous studies $[15,16]$, multi-depth branching microchannel networks were laser machined into silicon with nanosecond (ns) ablation followed by acid etching. However, the channel depth range with a reasonable surface quality was not wide enough to realize various microchannel network designs. Although the chemical wet etching post-processing smoothes the surface roughness, the relatively long chemical etching time required to remove debris buildup, resulting from a strong thermal reaction of irradiated silicon, limits the minimum channel size. Alternatively, fs ablation provides better surface quality and resolution than ns ablation by minimizing the thermal impact on irradiated material $[1,2]$.
There have been extensive studies on fs ablation of silicon [17-24]. Generally, the repetition rates $(<$ several $\mathrm{kHz}$ ) of the fs pulses used in these studies are accompanied by low processing speed. This is because relatively low pulse fluence is often required for high processing qualities, which is not wide enough to compensate the productivity loss by such a low repetition rate [24]. In the majority of applications including our artificial lung fabrication, however, macroscopic amounts of material removal and micron-level precision are required. Therefore, the development of silicon micromachining processes with high repetition rate $(200 \mathrm{kHz})$ fs pulses would have significant impact. The machining range, the processing speed, and the machined quality are all considered in this study.

Achieving the necessary feature depth with quality surface and rapid processing speed requires efficient energy transfer from laser to the target; however, the energy transfer must be well controlled in order to maintain sufficient surface smoothness. The laser energy transferred to the target can be manipulated with processing parameters such as the pulse energy, the focal position, the scan speed, and the number of scan passes. Due to the high repetition rate of the fs laser used in this study, the number of scan passes and the scan speed are manipulated as two main parameters. First, the ablation depth range in a single pass scanning is measured to optimize the pulse energy, the focal position, and the scan speed. In multiple-pass scanning, the influence of the scan speed on the channel depth, the material removal rate (MRR), and the surface roughness is studied.

As a demonstration, a 9-level branching network obeying Murray's law, for which the depth range is $50-320 \mu \mathrm{m}$, is laser machined into a silicon wafer. Actual branching networks are created out of polydimethylsiloxane (PDMS) using the laser machined silicon structures as molds.

\section{Experimental method}

The schematic of the fs laser micromachining setup is shown in figure 2. The laser used for this study is a prototype fiber laser operating at $1040 \mathrm{~nm}$. The laser is set to produce $\sim 600$ fs pulses with $\sim 10 \mu \mathrm{J}$ pulse energy at a repetition rate of $200 \mathrm{kHz}$. So the output average power is $\sim 2 \mathrm{~W}$ at $200 \mathrm{kHz}$. The average power is varied using a combination of a halfwave plate and a polarizer in beam delivery. A quarter-wave 


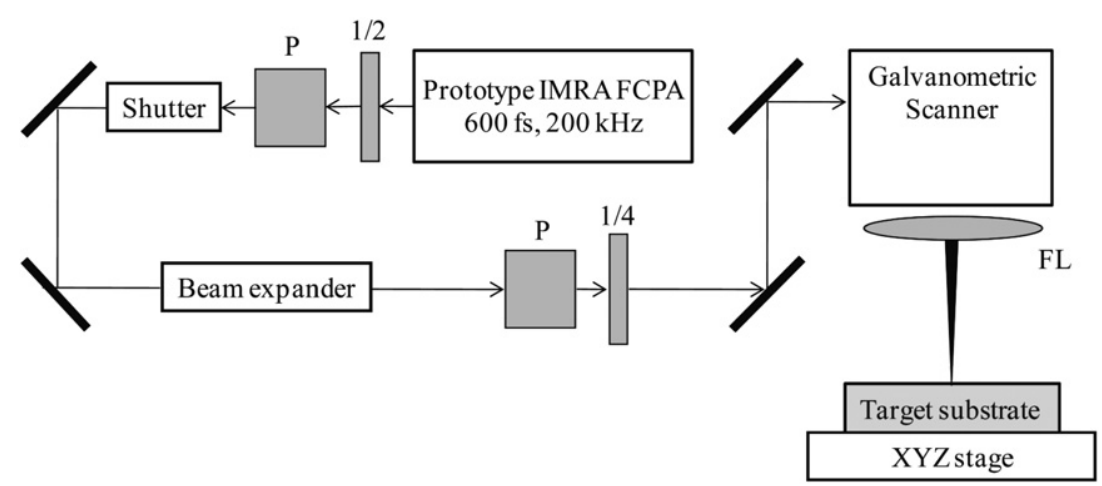

Figure 2. Experimental system schematic: polarizer $(P)$, half wave plate $(1 / 2)$, quarter wave plate (1/4), focal lens (FL).

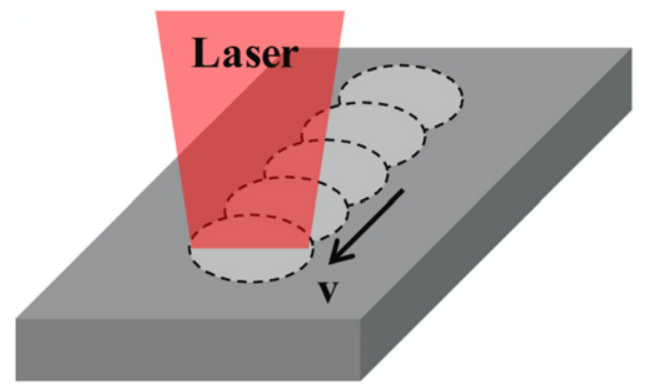

Figure 3. Single pass laser scan with a scan speed $v$.

plate is used to circularly polarize the beam at target. The beam shape is near Gaussian. The beam spot size of full width $1 / \mathrm{e}^{2}$ maximum $\left(\mathrm{FW} 1 / \mathrm{e}^{2} \mathrm{M}\right)$ is determined from the curve fitting of the dependence of the radii of the ablated spots on the pulse energy [25]. Using a $125 \mathrm{~mm}$ focal length F-theta objective, the beam spot size at focus is about $22 \mu \mathrm{m}$ in diameter. A two-mirror galvanometric scanner and an $X-Y-Z$ motion stage are used to control the position and the motion of laser beam spots. For wide channels, the beam is scanned side by side with $20 \mu \mathrm{m}$ pitch. Channels are machined into the silicon wafer with a $300 \mathrm{~nm}$ thick nitride coating. The wafer is N-type with $\left\langle\begin{array}{lll}1 & 0 & 0\end{array}\right)$ crystal orientation. As post-processing, chemical wet etching with a solution of $49 \% \mathrm{HF}$ and $69 \% \mathrm{HNO}_{3}$ with a volume ratio of 10:1 is done to smooth the roughness of the laser-ablated surface [15]. The nitride layer is resistive to this etchant, so the laser-ablated area is selectively etched. SEM images of the laser-ablated surface are used to measure the size of microchannels. For the cross-section images of microchannels, laser machined silicon samples are cleaved along cleavage planes, to which channels are machined to be perpendicular, after scoring with a glass cutter. Surface roughness is measured with a commercial surface profiler (Taylor Hobson: Intra). Each value is an average of three experimental measurements. In the measurement of channel size, absolute deviations are less than $5 \%$.

\section{Results and discussion}

\subsection{Single pass scan}

In the laser scan with a single pass as illustrated in figure 3, the pulse energy $(\Delta E)$, the focal position $(z)$, and the scan speed $(v)$ are varied to control the microchannel depth $\left(D_{\mathrm{mc}}\right)$. Figure 4(a) shows a SEM image of a fs-ablated channel with $\Delta E=1.14 \mu \mathrm{J}$ at $v=5 \mathrm{~mm} \mathrm{~s}^{-1}$ and $z=0.4 \mathrm{~mm}$ following $20 \mathrm{~s}$ acid etching. This channel has a depth of $D_{\mathrm{mc}} \sim$ $24 \mu \mathrm{m}$ and width of $\sim 50 \mu \mathrm{m}$. The laser ablation depth ( $\left.D_{\text {la }}\right)$ is smaller than $D_{\mathrm{mc}}$ by the depth etched by the acid solution. The difference is estimated to be approximately $17 \mu \mathrm{m}$. While its machined quality proves to be superior to that of longer pulses, fs pulse interaction with silicon still leads to considerable surface roughness [18, 19, 21-24]. As shown in figure 4(a), the acid etching effectively smoothes out relatively minor roughness problem due to debris buildup or laserinduced periodic surface structures (LIPSS) [22]. However, the notch-like defect on the bottom of channels shown in figure $4(b)$ is retained even after acid etching. Such a defect may be created by the discrepancy between the beam penetration depth and the actual material removal depth. Slower scan provides more time for laser energy to penetrate into silicon; however, this increased penetration does not necessarily result in more material removal as excessive overlap of high-intensity pulses can result in the interaction of the laser pulse with the ablation debris rather than the desired target. By selecting appropriate scan speeds, the defect can be avoided to achieve smooth surface and effective depth control. The value is chosen as the minimum value at which the defect is not formed, so that depth is maximized with no defects. To select optimized $v$, single pass channels are created for the scan speeds varying from 1 to $30 \mathrm{~mm} \mathrm{~s}^{-1}$ by $1 \mathrm{~mm} \mathrm{~s}^{-1}$ increment with $9.72 \mu \mathrm{J}\left(2.56 \mathrm{~J} \mathrm{~cm}^{-2}\right)$ pulses at the repetition rate $(f)$ of $200 \mathrm{kHz}$. It is observed that when the scan speed is set to $5 \mathrm{~mm} \mathrm{~s}^{-1}$, the notch-like shape is not observed after $20 \mathrm{~s}$ acid etching. With the optimized scan speed found above, it is found that $D_{\mathrm{mc}}$ variation from 17 to $63 \mu \mathrm{m}$ can be achieved by manipulating the pulse energy and the focal position below the surface. This is shown in figure 4(c). The minimum depth, $17 \mu \mathrm{m}$, is the result of the laser removal of nitride coating only and subsequent $20 \mathrm{~s}$ acid etching. Thus, using single pass scanning at focus with $2.56 \mathrm{~J} \mathrm{~cm}^{-2}$ pulse fluence and $v=5 \mathrm{~mm} \mathrm{~s}^{-1}$, the ablation depth is $D_{\mathrm{la}} \sim 46 \mu \mathrm{m}$. Higher scan speed results in reduced $D_{\text {la }}$ for single pass scan as reported in many other papers [18, 22, 24]. Using an effective number of pulses for single pass scan driven by Crawford et al [22], the ablation depth per pulse (adpp) is estimated to be $\sim 84 \mathrm{~nm}$. Crawford et al [22] performed a systematic study 


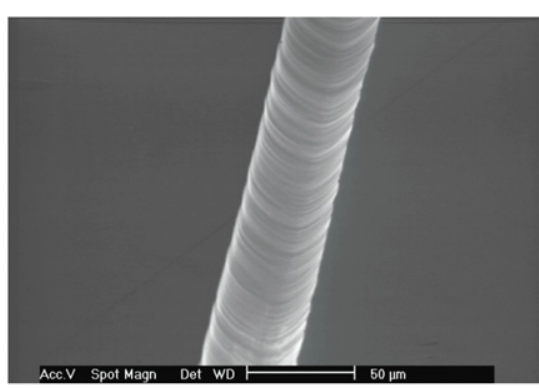

(a)

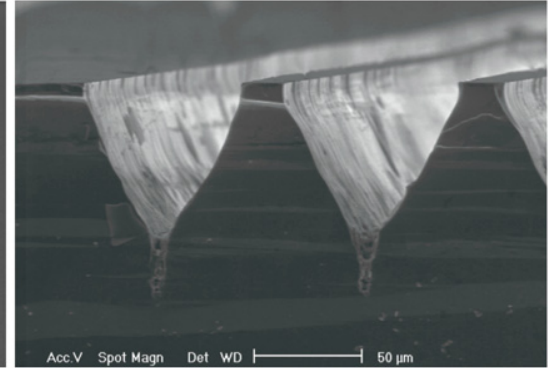

(b)

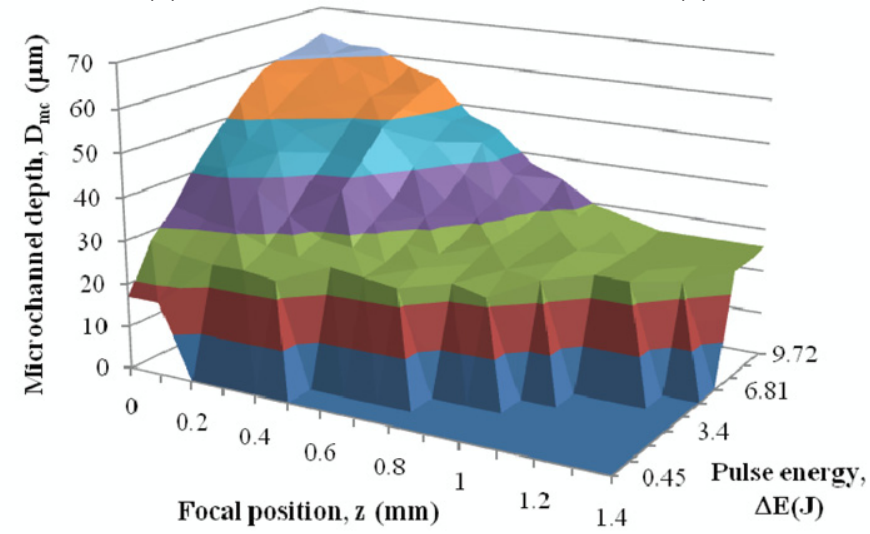

(c)

Figure 4. SEM images of fs-ablated channels on nitride coated silicon at the scan speed of (a) $5 \mathrm{~mm} \mathrm{~s}^{-1}(\Delta E=1.14 \mu \mathrm{J}, z=0.4 \mathrm{~mm})$ and (b) $1 \mathrm{~mm} \mathrm{~s}^{-1}(\Delta E=9.72 \mu \mathrm{J}, z=0.0 \mathrm{~mm})$ after $20 \mathrm{~s}$ chemical etching. (c) Depth of laser-ablated channel versus focal position below the surface and average power at $5 \mathrm{~mm} \mathrm{~s}^{-1}$ scan speed. Depths are measured after $20 \mathrm{~s}$ chemical etching.

on the fabrication of linear grooves in $\left\langle\begin{array}{lll}1 & 0 & 0\end{array}\right)$ silicon using 150 fs pulses at $f=1 \mathrm{kHz}$ and the wavelength of $800 \mathrm{~nm}$. The ablation depth per pulse is measured in terms of the fluence ranging from $0.1 \sim 11 \mathrm{~J} \mathrm{~cm}^{-2}$ for $v=0.1,0.25$, and $0.5 \mathrm{~mm} \mathrm{~s}^{-1}$. The value increases up to adpp $\sim 1 \mu \mathrm{m}$ with the fluence of $11 \mathrm{~J} \mathrm{~cm}^{-2}$. The fluence of $\sim 3 \mathrm{~J} \mathrm{~cm}^{-2}$ resulted in adpp of $100 \sim 170 \mathrm{~nm}$ depending on $v$. The result is similar in magnitude to ours, although their wavelength, pulse duration, and experimental conditions are different. Although our $v=$ $5 \mathrm{~mm} \mathrm{~s}^{-1}$ is higher by an order of magnitude, the pulse-topulse distance $(v / f)$ is shorter by an order of magnitude due to the high repetition rate $(200 \mathrm{kHz})$. Less spacing between pulses can lead to more roughness and debris, but acid etching used in our study allows a shorter pulse-to-pulse distance while maintaining good surface quality. The productivity can be estimated by calculating $f$-adpp. For the same fluence, therefore, the productivity of our laser setup at $f=200 \mathrm{kHz}$ would be higher by approximately two orders of magnitude than that of the study at $f=1 \mathrm{kHz}$. The productivity of the low repetition rate fs pulses can be increased by increasing $\Delta E$. However, it is known that high $\Delta E$ is accompanied with a quality loss. An optimal fluence range of fs pulses for quality machining of silicon is suggested by several groups [21, 24], and it is reported that the ablation depth is limited at very high $\Delta E$ [20]. Therefore, high $f$ with low $\Delta E$ is recommended to improve the productivity and to minimize the quality loss.

The width of single pass channel $\left(W_{s}\right)$ varies from 35 to $62 \mu \mathrm{m}$ as $\Delta E$ increases from 0.45 to $9.2 \mu \mathrm{J}$ when machined at focus followed by $20 \mathrm{~s}$ etching. The width of single pass

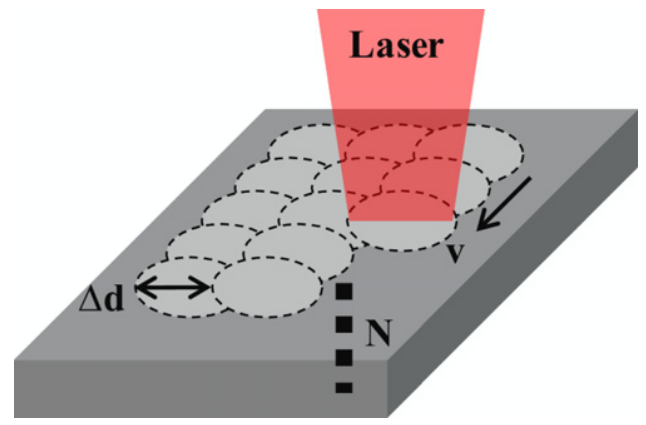

Figure 5. Multiple-pass laser scan: each layer is scanned parallel with the pitch, $\Delta d$, of $20 \mu \mathrm{m}$, and the number of laser scanned layers, $N$, is manipulated to control the microchannel depth.

channel is further increased up to $95 \mu \mathrm{m}$ as the $9.2 \mu \mathrm{J}$ beam is defocused up to $1 \mathrm{~mm}$ at $5 \mathrm{~mm} \mathrm{~s}^{-1}$ scan speed. The effect of $v$ on the width is relatively small compared to $\Delta E$ and $z$.

\subsection{Multiple-pass scan}

To increase the range of the microchannel depth $\left(D_{\mathrm{mc}}\right)$ and the width $(W)$, multiple-pass scan is used. As illustrated in figure 5 , this process is a layer-by-layer ablation process. To vary the channel width, multiple parallel lines are scanned with a separation $(\Delta d)$ of $20 \mu \mathrm{m}$ so that the number of lateral parallel lines $\left(N_{w}\right)$ determines the width of the channel (resolution is determined by $\Delta d$ and $W_{s}$ ). The number of laserscanned layers $(N)$ is manipulated to control $D_{\mathrm{mc}}$; however, the ablation depth does not always increase with $N$ as observed in 

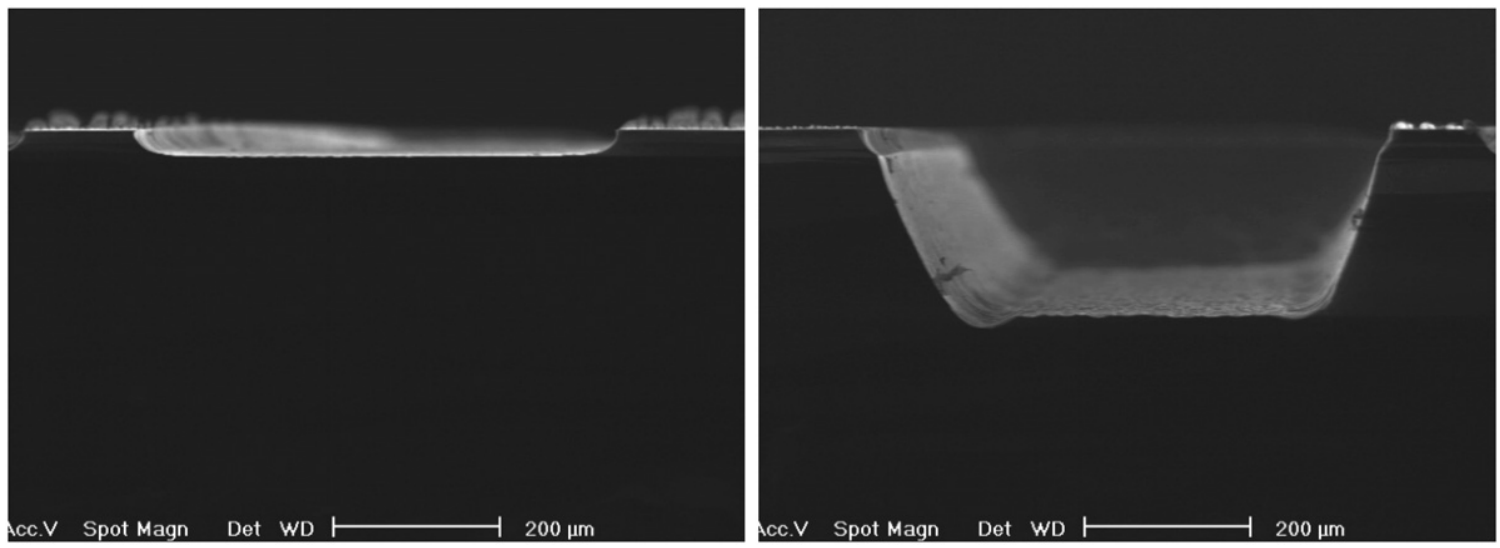

Figure 6. SEM images of the cross section of laser machined channels onto silicon after $20 \mathrm{~s}$ acid etching. The scan speed is $480 \mathrm{~mm} \mathrm{~s}$.

several studies [22, 24]. The depth limit (or saturation) after several laser scans can be explained by many reasons including competition between ablation and material redeposition [22], and formation of a cone-shaped spike array [24]. As shown in figure 6, we are able to create the channel depth, $D_{\mathrm{mc}}$, from 20 $\mu \mathrm{m}$ to several hundred $\mu \mathrm{m}$ with $9.72 \mu \mathrm{J}$ pulses by varying $N$. Figure 7( $a$ ) shows $D_{\mathrm{mc}}$ (measured after $20 \mathrm{~s}$ acid etching, which adds $\sim 17 \mu \mathrm{m}$ depth to the ablation depth) as a function of $N$ for $v=30,120,480$, and $1920 \mathrm{~mm} \mathrm{~s}^{-1}$. Here, an appropriate choice of $v$ is important to achieve a wide machining range. For example, with $v=5 \mathrm{~mm} \mathrm{~s}^{-1}, D_{\mathrm{mc}}$ does not increase with $N$. This may be due to the mixing of resolidified molten silicon and debris which cover the surface and block laser energy delivery to the target surface. It shows that such redeposition does not decrease with $N$ at $v=5 \mathrm{~mm} \mathrm{~s}^{-1}$. In the previous single pass scan, such a problem is not observed even with the same operating condition. Unlike the single pass scan, in the multiple parallel scan, each scan line is overlapped according to the pitch. So this overlap causes the redeposition which is thought to result from heat accumulation [26-30]. However, less redeposition is observed as the scan speed increases. At $30 \mathrm{~mm} \mathrm{~s}^{-1}$, the ablation depth saturates to around $300 \mu \mathrm{m}$ after $N \sim 50$ due to the debris buildup and growth of conical spikes. For $120 \mathrm{~mm} \mathrm{~s}^{-1}$, however, such saturation is not observed up to $N=260$ corresponding to $\sim 450 \mu \mathrm{m}$ ablation depth. The number of scanned layers that can be machined before redeposition starts to accumulate is observed to increase with $v$. The ablation depth with $N=95$ and $v=100 \sim 1900 \mathrm{~mm} \mathrm{~s}^{-1}$ in $100 \mathrm{~mm} \mathrm{~s}^{-1}$ increment is shown in figure $7(b)$. In the measurement, the effect of the acid etching is not included. The data fit well to a $D_{\text {la }}=v^{-0.853}$ in contrast to inversely proportional decay of single pass scan $[18,22]$ and exponential decay behavior of multiple pass [24]. Previous studies used the Ti:sapphire laser system with 150 fs pulses at $1 \mathrm{kHz}$ and their fitting ranges are much narrower than ours: their ranges of the depth and $v$ are less than $40 \mu \mathrm{m}$ and $10 \mathrm{~mm} \mathrm{~s}^{-1}$, respectively. Therefore, a quantitative direct comparison may be not appropriate. Similar to the single pass scan, our $v>100 \mathrm{~mm} \mathrm{~s}^{-1}$ for multiple-pass scan is higher by an order of magnitude, but the pulse-to-pulse distance $(v / f)$ is shorter by an order of magnitude due to the high repetition rate $(200 \mathrm{kHz})$.
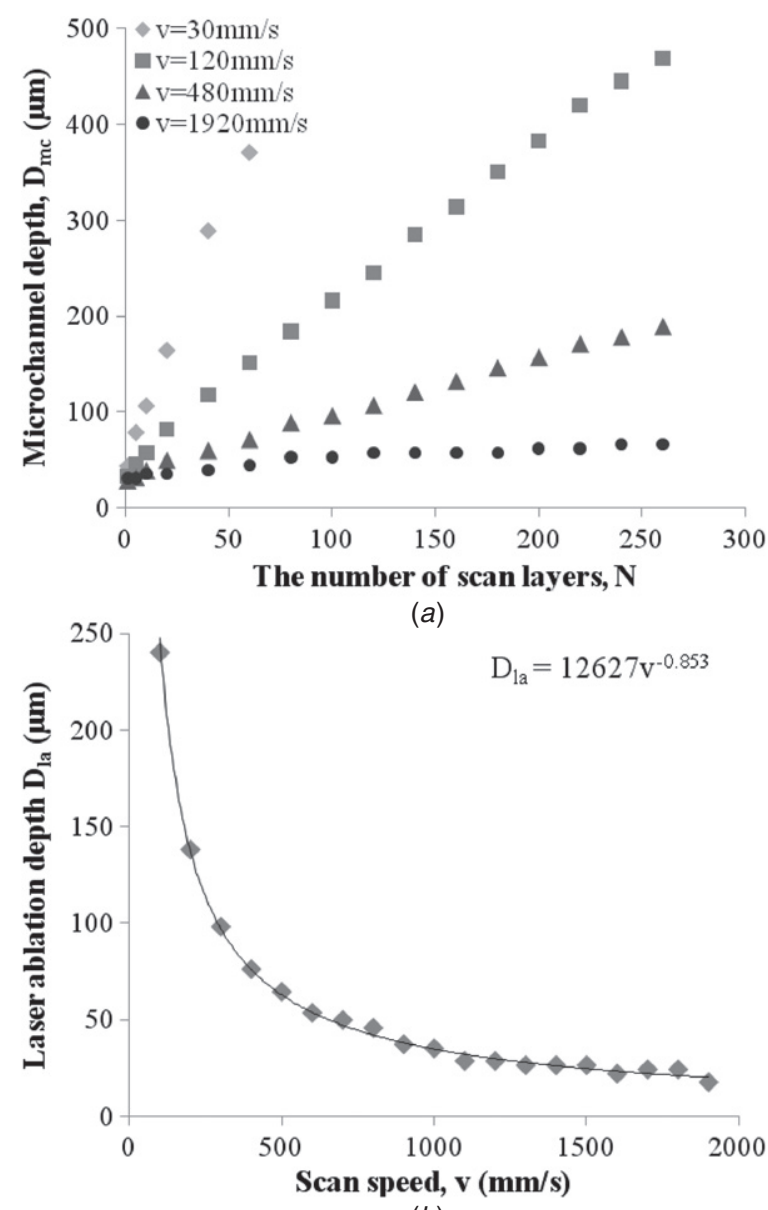

(b)

Figure 7. (a) Ablation depth into nitride coated silicon wafer versus the number of scan passes, $N$, after $20 \mathrm{~s}$ acid etching. $9.72 \mu \mathrm{J}$ pulses are scanned at focus with $v=30,120,480$, and $1920 \mathrm{~mm} \mathrm{~s}^{-1}$. (b) The ablation depth versus $v$ with $N=95$ and $2.56 \mathrm{~J} \mathrm{~cm}^{-2}$ fluence at focus.

$v$ also plays a key role in the laser processing speed and the machined surface quality. The variation of the MRR with $v$ is presented in figure $8(a)$. The MRR is measured for $N=95,195$, and 395. In the measurement, the effect of the acid etching is not included. The maximum MRR $\sim 0.5 \mathrm{~mm}^{3} \mathrm{~min}^{-1}$ is obtained at $v \sim 750 \mathrm{~mm} \mathrm{~s}^{-1}$. 


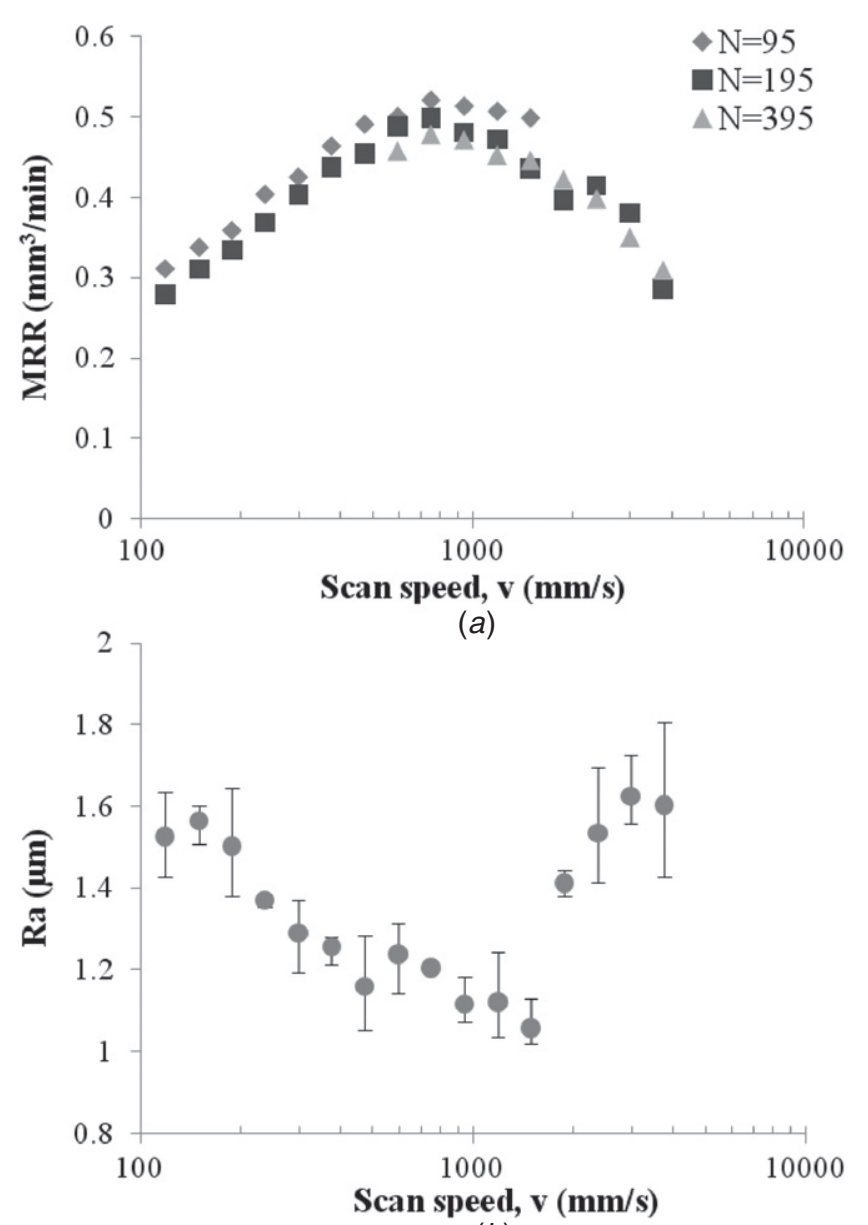

(b)

Figure 8. $(a)$ MRR versus scan speed $v$ and $(b)$ the average surface roughness $R a$ versus the scan speed $v$ after $24 \mathrm{~s}$ etching. Each $R a$ value is an average of three experimental measurements.

Figure 9 shows the corresponding overlap of craters for $v=1200,2400$, and $4800 \mathrm{~mm} \mathrm{~s}^{-1}$. Based on the figure, it is expected that $v=750 \mathrm{~mm} \mathrm{~s}^{-1}$ corresponds to $\sim 84 \%$ overlap of craters with $\sim 24 \mu \mathrm{m}$ diameter created by a $9.72 \mu \mathrm{J}$ $\left(2.56 \mathrm{~J} \mathrm{~cm}^{-2}\right)$ pulse. Lee et al [24] machined microchannels in silicon using a Ti:sapphire laser operating at $775 \mathrm{~nm}$ with the pulse duration of $150 \mathrm{fs}$ at $1 \mathrm{kHz}$. They found the optimal fluence range for the best surface quality to be $2-8 \mathrm{~J} \mathrm{~cm}^{-2}$. Using $6.26 \mathrm{~J} \mathrm{~cm}^{-2}$ fluence, they obtained the ablation depth, $D_{\text {la }}$, of $\sim 60 \mu \mathrm{m}$ with the pitch, $\Delta d=2 \mu \mathrm{m}$ and the number of scan layers, $N=20$. Assuming that the cross section of a channel machined with the multiple pass is rectangular, the MRR can be derived as [31]

$$
\mathrm{MRR} \sim \frac{D_{\mathrm{la}} \cdot \Delta d \cdot v}{N}
$$

Using this equation, we can estimate that the MRR is $\sim 0.003 \mathrm{~mm}^{3} \mathrm{~min}^{-1}$. Therefore, our MRR $\left(\sim 0.5 \mathrm{~mm}^{3} \mathrm{~min}^{-1}\right)$ is higher by approximately two orders of magnitude than that of Lee et al. This is expected because we used a fraction of their fluence (for example, $2.56 \mathrm{~J} \mathrm{~cm}^{-2}$ compared to $6.26 \mathrm{~J} \mathrm{~cm}^{-2}$ ) and 200 times of their repetition rate. The MRR slightly decreases as $N$ increases as shown in figure 8(a). As mentioned above, this can be explained by the redeposition
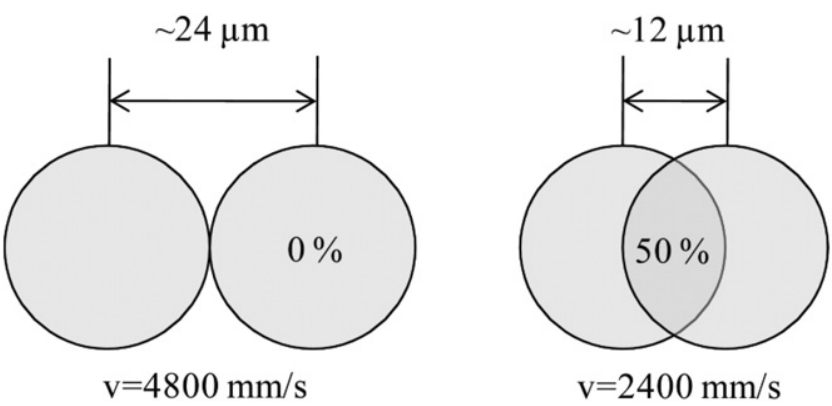

Figure 9. Schematic sketch to illustrate the relation between the scan speed and the overlap of each crater with a diameter of $\sim 24 \mu \mathrm{m}$. The interval between each pulse at $200 \mathrm{kHz}$ is $5 \mu \mathrm{s}$, so the scan speed is more than $\sim 4800 \mathrm{~mm} \mathrm{~s}^{-1}$ to separate each crater created by a single pulse.

or the formation of the conical spikes with $N$. Another reason might be the defocusing effect as the ablation depth increases, since the focal position is fixed during machining and for this case and the Rayleigh range is $\sim 300 \mu \mathrm{m}$. Figure $8(b)$ shows the average surface roughness $(R a)$ of the same ablation depth in terms of $v$. Here, the depths of channels are fabricated to $100 \mu \mathrm{m}$ with less than $2 \%$ error by varying $N$ according to $v$ and $R a$ is measured after acid etching for $24 \mathrm{~s}$. The dependence of $R a$ on $v$ is similar to that of the MRR. Thus, $v$, which provides the optimal material removal, also creates a smooth channel surface. This is described by the relation between heat accumulation and overlap of fs pulses at a high repetition rate [26-30]. At low $v$, the distance between pulses is close so that the thermal effect on the surface is increased by the heat accumulation. The thermal reaction is accompanied by debris buildup. This debris fills the channel and blocks laser energy delivery to the target surface increasing the surface roughness. As $v$ is increased, debris buildup is reduced which increases the overall MRR and reduces $R a$. However, as $v$ is further increased, the effectiveness of material removal is reduced due to the increased pulse-to-pulse distance. This results in higher roughness because of increased peak-to-valley roughness. These competing processes create an optimal MRR and $R a$ at certain $v$ as shown in figure 8 .

$R a$ as a function of $D_{\mathrm{mc}}$ is plotted in figure 10. For fs ablation, $v$ is set to $480 \mathrm{~mm} \mathrm{~s}^{-1}$ and $N$ is varied from 1 to 330 resulting in $D_{\mathrm{mc}}$ from 28 to $236 \mu \mathrm{m}$ after $20 \mathrm{~s}$ etching. For comparison, channels are also machined with a pulsed Nd:YAG laser operating at $1064 \mathrm{~nm}$ with $500 \mathrm{~Hz}$ repetition rate and $100 \mu$ s pulse width [15]. The $100 \mu$ s pulses are modulated to produce a series of $200 \mathrm{~ns}$ pulses with $10 \mu \mathrm{s}$ interval. The near-Gaussian beam spot size of $F W 1 / \mathrm{e}^{2} \mathrm{M}$ is about $30 \mu \mathrm{m}$ in diameter. The average power is varied from 0.2 to $3 \mathrm{~W}$ with the scan speed of $8 \mathrm{~mm} \mathrm{~s}^{-1}$ and $0.5 \mathrm{~mm}$ defocus below surface. For both fs and ns laser ablation, the surface roughness increases with the ablation depth as observed in many laser ablation studies [18, 19, 24]. For $20 \mathrm{~s}$ etch time, the maximum ns ablation depth, with $R a$ of $<2 \mu \mathrm{m}$, is $\sim 50 \mu \mathrm{m}$, whereas, for fs ablation, a depth of more than $200 \mu \mathrm{m}$ can be achieved with $R a<2 \mu \mathrm{m}$. When the etch time for the ns-ablated channel is increased to $40 \mathrm{~s}$, the maximum depth with $R a<2 \mu \mathrm{m}$ is increased to $\sim 130 \mu \mathrm{m}$; however, the minimum channel depth also increases to $\sim 90 \mu \mathrm{m}$. 


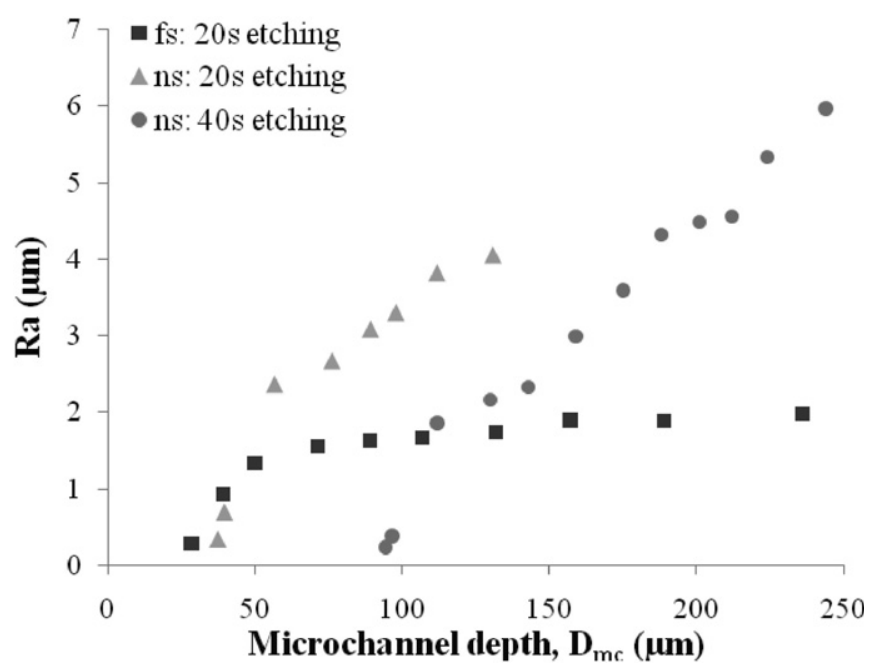

Figure 10. Average roughness $R a$ versus depth $D_{\mathrm{mc}}$ for fs and ns laser machined channels after acid etching. For fs ablation, the scan speed is set to $v=480 \mathrm{~mm} \mathrm{~s}^{-1}$.

\subsection{Laser machining of the multi-depth microchannel network}

Based on the above study, a 9-level branching network is laser machined onto a nitride coated silicon wafer with the fs laser, as shown in figure 11(a). The SEM image in figure 11(b) shows the surface morphology of the laser machined channel surface. The network is designed to bifurcate according to Murray's law and thus the channels become shallower and narrower at each bifurcation as illustrated in figure 1 . The depth of the branching network ranges from 50 to $317 \mu \mathrm{m}$ as presented in table 1. Here, the depth-to-width ratio of each generation remains approximately constant as well as the ratio of the depth to the length. In addition, all paths from inlet to outlet of the network are designed to have the same length to achieve the same pressure drop. To control the depth and the width of each generation of channels, $N$ and $N_{w}$ are varied. $N$ for desired depths is chosen by interpolating the plot in figure $7(a)$. The laser processing of this branching network
Table 1. Depths $\left(D_{\mathrm{mc}}\right)$ and widths $(W)$ of each generation of a 9-level branching network and corresponding number scan layers $(N)$ and number of lateral parallel lines $\left(N_{w}\right)$ with 20 s etching. Hydraulic diameters $\left(D_{h}\right)$ are estimated assuming that the channel cross sections are rectangular. The pulse energy is $9.72 \mu \mathrm{J}$ and the scan speed is $480 \mathrm{~mm} \mathrm{~s}^{-1}$.

\begin{tabular}{lllllr}
\hline Generation & $D_{\mathrm{mc}}(\mu \mathrm{m})$ & $W(\mu \mathrm{m})$ & $D_{h}(\mu \mathrm{m})$ & $N$ & $N_{w}$ \\
\hline First & 317 & 3180 & 577 & 462 & 157 \\
Second & 252 & 2520 & 458 & 356 & 124 \\
Third & 200 & 2000 & 364 & 271 & 98 \\
Fourth & 159 & 1600 & 289 & 204 & 78 \\
Fifth & 126 & 1260 & 299 & 150 & 61 \\
Sixth & 100 & 1000 & 182 & 108 & 48 \\
Seventh & 79 & 800 & 143 & 74 & 38 \\
Eighth & 63 & 640 & 115 & 47 & 30 \\
Ninth & 50 & 500 & 91 & 26 & 23 \\
\hline
\end{tabular}

took about $5 \mathrm{~h}$. So macroscopic material removal in addition to micron level precision is achieved with fs ablation. A final blood flow/oxygen-exchange (oxygenator) device is created out of PDMS using the laser machined silicon structure as a mold as shown in figure 11( $a$ ) according to the procedure described in [15]. Then, a thin film of PDMS is placed on top of the PDMS network to make a closed channel. To function as an artificial lung, blood flows inside the channels and gases are exchanged through the gas permeable PDMS film.

\section{Conclusions}

Femtosecond (fs) ablation of microchannels in silicon is studied to develop branching networks to serve gas exchangers for use in artificial lungs. Wide ranges of microchannel size with smooth surface are required to realize artificial vascular network simulating the mammalian respiratory blood vessel tree obeying Murray's law. In the development of such devices, the total fabrication time is also a significant consideration. Using a high repetition rate significantly increases the fabrication speed of fs pulses. We show that the expansion of the machining range with smooth surface and the increase of processing speed in a large part rely on

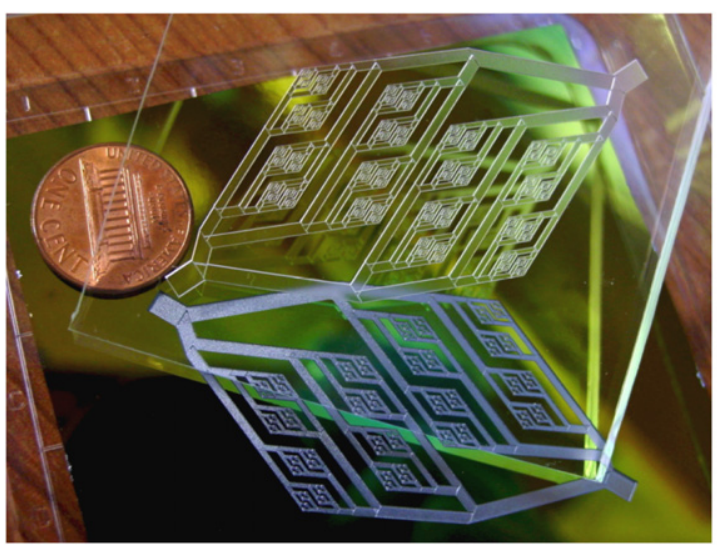

(a)

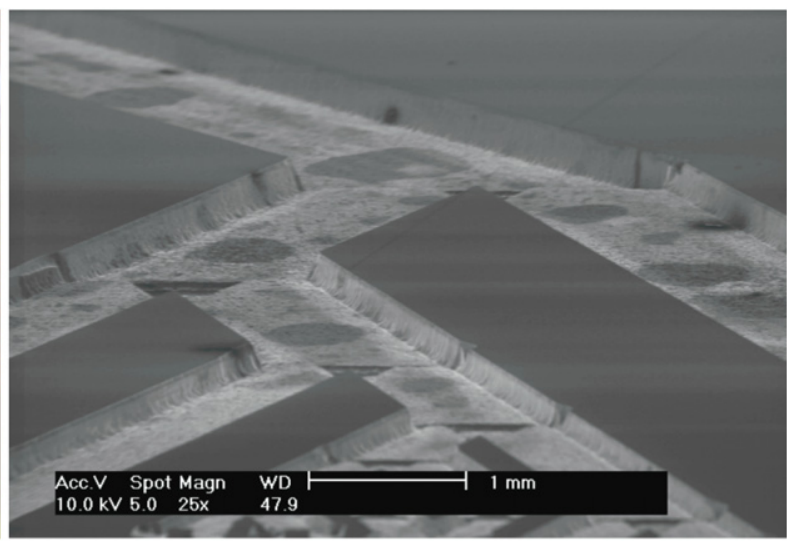

(b)

Figure 11. (a) 9-level branching network laser machined onto nitride coated silicon and PDMS replica. The process is done at focus with $480 \mathrm{~mm} \mathrm{~s}^{-1}$ scan speed and the etching time is $20 \mathrm{~s}$. (b) SEM image of the network in silicon. 
the appropriate choice of the scan speed. Conveniently, we find that the optimal scan speed for effective material removal also produces the smoothest ablation surface. Utilizing a high repetition rate of $200 \mathrm{kHz}$ fs laser system with $\sim 10 \mu \mathrm{J}$ pulse energy, the channel depth varies up to several hundreds of microns with $R a<2 \mu \mathrm{m}$ and MRR $\sim 0.5 \mathrm{~mm}^{3} \mathrm{~min}^{-1}$. A 9-level microchannel network is realized as a demonstration. Therefore, the laser technique developed in this study enables the fabrication of microchannels mimicking the feature of the natural vasculature as needed for the development of artificial lungs. The mimicked physiological features are expected to contribute to the further development of artificial lungs. In addition to the artificial lung development, the simplicity and flexibility of our laser technique are beneficial to the developments of MEMS, lab-on-a-chip systems, and other biotechnology applications.

\section{Acknowledgment}

This work was sponsored by IMRA America Inc. as a member of the National Science Foundation (NSF) of Industry University Cooperative Research Program (I/UCRC) for Lasers and Plasmas in Advanced Manufacturing.

\section{References}

[1] Stuart B C, Feit M D, Herman S, Rubenchik A M, Shore B W and Perry M D 1996 Nanosecond-to-femtosecond laser-induced breakdown in dielectrics Phys. Rev. B 53 1749-61

[2] Chichkov B N, Momma C, Nolte S, von Alvensleben F and Tünnermann A 1996 Femtosecond, picosecond, and nanosecond laser ablation of solids Appl. Phys. A 63 109-15

[3] Giridhar M S, Seong K, Schülzgen A, Khulbe P, Peyghambarian N and Mansuripur M 2004 Femtosecond pulsed laser micromachining of glass substrates with application to microfluidic devices Appl. Opt. 43 4584-9

[4] Hwang D J, Choi T Y and Grigoropoulos C P 2004 Liquid-assisted femtosecond laser drilling of straight and three-dimensional microchannels in glass Appl. Phys. A 79 605-12

[5] Sugioka K, Cheng Y and Midorikawa K 2005 Three-dimensional micromachining of glass using femtosecond laser for lab-on-a-chip device manufacture Appl. Phys. A 81 1-10

[6] Zwischenberger J B, Anderson C M, Cook K E, Lick S D, Mockros L F and Bartlett R H 2001 Development of an implantable artificial lung: challenges and progress $A S A I O$ J. 47 316-20

[7] Zwischenberger J B and Alpard S K 2002 Artificial lungs: a new inspiration Perfusion 17 253-68

[8] Haft J W, Griffith B P, Hirschl R B and Bartlett R H 2002 Results of an artificial-lung survey to lung transplant program directors J. Heart Lung Transplant. 21 467-73

[9] Matheis G 2003 New technologies for respiratory assist Perfusion 18 245-51

[10] Wegner J A 1997 Oxygenator anatomy and function J. Cardiothorac. Vasc. Anesth. 11 275-81
[11] Murray C D 1926 The physiological principle of minimum work Proc. Natl Acad. Sci. 12 207-14

[12] Murray C D 1926 The physiological principle of minimum work applied to the angle of branching of arteries Proc. Natl Acad. Sci. 12 835-41

[13] Huang W, Yen R T, McLaurine M and Bledsoe G 1996 Morphometry of the human pulmonary vasculature $J$. Appl. Physiol. 81 2123-33

[14] Emerson D R, Cieślicki K, Gu X and Barber R W 2006 Biomimetic design of microfluidic manifolds based on a generalized Murray's law Lab Chip 6 447-54

[15] Kam D H and Mazumder J 2008 Three-dimensional biomimetic microchannel network by laser direct writing J. Laser Appl. 20 185-91

[16] Kam D H, Shah L and Mazumder J 2008 Laser micromachining of branching networks Proc. SPIE $\mathbf{6 8 8 0} 68800$ S. $1-$ S.8

[17] Momma C, Nolte S, Chichkov B N, von Alvensleben F and Tünnermann A 1997 Precise laser ablation with ultrashort pulses Appl. Surf. Sci. 109/110 15-9

[18] Ameer-Beg S, Perrie W, Rathbone S, Wright J, Weaver W and Champoux H 1998 Femtosecond laser microstructuring of materials Appl. Surf. Sci. 127-129 875-80

[19] El-Bandrawy M and Gupta M C 2003 Femtosecond laser micromachining of silicon for MEMS Proc. SPIE 4977 219-25

[20] Bärsch N, Körber K, Ostendorf A and Tönshoff K H 2003 Ablation and cutting of planar silicon devices using femtosecond laser pulses Appl. Phys. A 77 237-42

[21] Coyne E, Magee J, Mannion P and O'Connor G 2003 A study of femtosecond laser interaction with wafer grade silicon Proc. SPIE 4876 487-99

[22] Crawford T H R, Borowiec A and Haugen H K 2005 Femtosecond laser micromachining of grooves in silicon with $800 \mathrm{~nm}$ pulses Appl. Phys. A 80 1717-24

[23] Wang Y, Dai N, Li Y, Wang X and Lu P 2007 Ablation and cutting of silicon wafer and micro-mold fabrication using femtosecond laser pulses J. Laser Appl. 19 240-4

[24] Lee S, Yang D and Nikumb S 2008 Femtosecond laser micromilling of Si wafers Appl. Surf. Sci. 254 2996-3005

[25] Liu J M 1982 Simple technique for measurements of pulsed Gaussian-beam spot sizes Opt. Lett. 7 196-8

[26] Gamaly E G, Rode A V and Luther-davies B 1999 Ultrafast ablation with high-pulse-rate lasers: part I. Theoretical considerations J. Appl. Phys. 85 4213-21

[27] Eaton S M, Zhang H and Herman P R 2005 Heat accumulation effects in femtosecond laser-written waveguides with variable repetition rate Opt. Express 13 4708-16

[28] Gattass R R, Cerami L R and Mazur E 2006 Micromachining of bulk glass with bursts of femtosecond laser pulses at variable repetition rates Opt. Express 14 5279-84

[29] Eaton S M, Zhang H, Ng M L, Li J, Chen W-J, Ho S and Herman P R 2008 Transition from thermal diffusion to heat accumulation in high repetition rate femtosecond laser writing of buried optical waveguides Opt. Express 16 9443-58

[30] Sakakura M, Shimizu M, Shimotsuma Y, Miura K and Hirao K 2008 Temperature distribution and modification mechanism inside glass with heat accumulation during $250 \mathrm{kHz}$ irradiation of femtosecond laser pulses Appl. Phys. Lett. 93231112

[31] Kam D H 2009 PhD Thesis University of Michigan, Ann Arbor, p 50 